\title{
Statistical Data Analysis of Culture for Innovation using an Open Data Set from the Australian Public Service
}

\author{
Warit Wipulanusat*, Kriengsak Panuwatwanich, Rodney A. Stewart \\ Griffith University, Gold Coast, Australia \\ warit.wipulanusategriffithuni.edu.au
}

\begin{abstract}
Opportunities for replicating large data sets that have already been collected by government, and made available to the public to provide the possibility of statistical data analysis, are starting to emerge. This study examines the factor structure of ambidextrous culture for innovation. Survey data was extracted from the State of the Service Employee Census 2014 comprising 3,125 engineering professionals in Commonwealth of Australia departments. Data were analysed using exploratory factor analysis (EFA) and confirmatory factor analysis (CFA). EFA returned a two-factor structure explaining $61.1 \%$ of the variance of the construct. CFA revealed that a twofactor structure was indicated as a validated model $(\mathrm{GFI}=0.99$, AGFI $=0.98$, RMSEA $=0.05$, RMR $=0.02, \mathrm{IFI}=0.99, \mathrm{NFI}=0.99, \mathrm{CFI}=0.99$, and TLI $=$ 0.98). From the results, the two factors extracted as characterising ambidextrous culture for innovation were innovative culture and performance-oriented culture.
\end{abstract}

Keywords: Open data set, Culture for innovation, Exploratory Factor Analysis, Confirmatory Factor Analysis

\section{Introduction}

An effective government culture is one that focuses on shared norms and basic values pertaining to innovation in the work environment and reflecting innovative practices, procedures, policies and structures. In this study, the dimensionality of culture for innovation was analysed using an exploratory factor analysis (EFA) and confirmatory factor analysis (CFA). The inter-relationship between variables and the factor structure of their measure was analysed using EFA. Subsequently, CFA was used to test the fit of the model.

Appropriate variables can be identified using EFA. Additionally, the relationships among large numbers of variables in the most general form can be analysed using this method through explanations in terms of their common underlying dimensions [1]. A number of factors were retained in the construct based on results from the EFA together with a clear estimation of the factor structures to be used as the measures of this construct. To confirm the validity of the measurement scale, CFA was sequentially conducted. CFA is a theory-driven technique which tests the hypotheses for a factor structure. This determines the validity of theoretical structures by testing 
the causal links among variables [2, 3]. This served to strengthen the results of the EFA and support the recognised factor structures derived from the EFA process. Researchers can use CFA to evaluate the structure of factors, identify the dimensions of a construct, and consider whether particular patterns of loadings match the data [1].

\section{$2 \quad$ Literature Review}

\subsection{Ambidextrous Culture for Innovation}

The ambidextrous culture for innovation is classified into two sets of cultural features. The first type of organisational culture is an innovative culture where an organisation orients toward experimenting with new solutions by exploring new resources, breaking through existing norms, and valuing flexibility, adaptability, creativity, risk taking, and entrepreneurship. Such an innovative culture encourages employees to implement new services, new technologies for product development, new organisational routines and structures [4]. Cultivating employee orientation toward innovation may lead them to feel that the organisation is full of spirit, can manage uncertainty in the work environment, and reduces unfavourable consequences in the organisation. Innovation orientation is also likely to lead to truly innovative breakthroughs due to its emphasis on creativity. Lægreid et al. [5] assert that innovative cultures can ensure employees consistently perceive an innovative cultural orientation, thus serving as guidance when they face challenges affecting innovation outcomes.

The second type of ambidextrous culture for innovation stresses organisational performance orientation, in which organisational characteristics are categorised into three elements: (a) strongly developed goal orientation; (b) a focus on task performance; and (c) a strong emphasis on quality of service delivery. Such a performance-oriented culture is accepted to increase innovation in public sectors because governments need to respond to the demands of clients and citizens to achieve targets and performance evaluation processes. According to Lægreid et al. [5], public sectors which have strongly developed performance-oriented cultures are more likely to have an innovative culture and promote innovative activities compared to other public sectors.

\subsection{Open Data Set from the Australian Public Service}

Opportunities for replicating large data sets in public management studies are starting to emerge. An open data set is defined as data that has already been collected by public or private organisations, and made available to the public to provide the possibility of study replications [6]. There are many advantages of using open data sets. One major advantage is the extent of the data available from studies which are conducted on a large national scale and cover a broad geographic scope. In contrast, a researcher is likely to collect a smaller sample than an organisation would. The second advantage is the professional data collection skills of research experts in large 
organisations may not be readily available to smaller studies [7]. The third advantage comes in terms of expense and time savings. Collecting data for a national survey with a sample size of 1,500 to 2,000 can be very expensive with the cost potentially exceeding $\$ 300,000$ in most cases [8]. The design and distribution of the questionnaire, as well as collecting and inputting the data, can take a considerable amount of money and time. Finally, when data is collected on a national scale, the large sample size offered by national databases has a higher likelihood of representation of the population and can furnish the power needed to effect generalisations of the findings. This is true in the context of this research, where the open data sets provided the above advantages to examine innovation in public sectors.

However, while the advantages of using open data sets are very practical, the disadvantages have a tendency to be more conceptual. A major disadvantage is that the data has not been designed particularly to address the researchers own questions. Researchers are not involved in the creation of the questionnaire, the types of questions, or the measurement scales; therefore, the researcher is limited to only using the available data. The second disadvantage is that the data collection process was not administered by researchers who opt to use open data. As a result, identification of any bias encountered during the collection process may not be detected as the survey instrument was designed and tested by others. The third disadvantage consists of access to the data, as there may be restrictions and conditions.

Governments in industrialised countries are now surveying their employees to consider their views and attitudes about the workplace, management, and human resources. In Australia, the State of the Service Employee Census examines the views of APS employees on workplace issues such as leadership, learning and development, health and wellbeing, and job satisfaction. The large sample size surveyed in the State of the Service Employee Census and its widespread coverage of federal departments can be applied for the generalisability of federal bureaucracy results on a national scale, including the perspectives of engineering professions because it also reaches the desired population of engineers within the federal government. In this study the potential limitations of using open data sets do not apply for the State of the Service Employee Census. The data set is in the Australian public service domain and can be accessed to address the research questions in this study. Using the census data assures no eligible respondents have been excluded from the survey sample, removing sampling bias and reducing sample error. There were no restrictions to use the data set and it is compatible with SPSS with only basic data cleaning required. Therefore, the most recent State of the Service Employee Census 2014 was adopted to conduct the quantitative analysis.

From an Australian public sector perspective, Torugsa and Arundel [9] employed a sample of 4,369 federal employees from the State of the Service Employee Census 2011, to explore factors associated with complexity and examined how complexity affected innovation outcomes. Torugsa and Arundel [10] also used the census data to investigate the characteristics of innovation implemented at the workgroup level and evaluated the different dimensions of the most significant innovation in the work group. Thus, open data sets have been identified by researchers to be valuable sources of information to examine innovation in public sectors. 


\section{Methodology}

Data released by the Australian Public Service Commission (APSC) from the 2014 APS employee census was used for this study. This survey instrument was administered to gather data from civil servants in Commonwealth departments using an online survey. The State of the Service Employee Census 2014 targeted full-time, permanent civil servants of federal departments throughout the Federal Government and was collected from 12 May to 13 June 2014. The survey was distributed to a random sample of employees from federal departments with 100 or more employees throughout Australia. The survey was self-administered on a voluntary basis. Employees were invited to participate in the survey using email solicitation and a web-based questionnaire. An e-mail was sent to the participants with a link to the questionnaire, so respondents could access the survey at a time and location convenient to their situation.

This study draws on the perspectives of engineers as key informants. Of all respondents, 3,570 reported their type of work as Engineering and Technical Family. Cases where an entire section was left blank were eliminated from the sample as nonresponsive, leaving a total of 3,125 observations available for this study. The survey items were measured on a 5 point Likert scale (Strongly Disagree $=1$ and Strongly Agree = 5).

The quantitative analysis commenced with multivariate statistics to analyse the data using the Statistical Package for the Social Sciences (SPSS) version 22 software. To clean the data and decrease systematic errors, missing values, outliers, and distribution of all measured variables were examined. This data screening was conducted to ensure there would be no corrupted data which could affect the accuracy of the estimation in the subsequent analysis [11].

\section{Results}

\subsection{Exploratory Factor Analysis}

To estimate the validity of the scales, exploratory factor analysis (EFA) was conducted to reduce the large number of variables into a smaller, more controllable set of dimensions $[1,2]$ and to summarise the data prior to including them into confirmatory factor analysis. The EFA is a multivariate statistical technique used to search for structure among a set of observed variables (i.e. questions in the questionnaire) and subsequently group highly inter-correlated observed variables into a latent factor. This approach allows researchers to be scientifically confident that the observed variables reproduce one single variable as a latent variable. Generally, the EFA can be performed by employing either R-type or Q-type factor analysis. The former identifies a set of dimensions that is latent in a large set of items, while the latter condenses large numbers of people into distinctly separate groups [1]. The Rtype was adopted in this study because the main purpose of this stage was to allocate the observed variables into a set of new composite dimensions (factors). 
According to Russell [12], the EFA must be conducted to determine whether a theoretical construct is a single or multidimensional factor, and to give a clear estimation of the factor structure of the measures. As a preliminary analysis, the EFA is very useful in the absence of an adequately detailed theory about the relations of variables to the underlying constructs [2]. Although all measured variables in the construct were derived from a comprehensive literature review and prior studies, the EFA was deemed necessary because these variables had not been operationalised extensively within the public sector context. Therefore, given that the model construct was measured by an independent scale, the EFA was conducted separately for the model construct to see if the chosen variables loaded on the expected latent factors were adequately correlated and, met the criteria of reliability and validity within this sample.

In order to assess the factorability of the data and ensure sampling adequacy, Bartlett's test of sphericity and the Kaiser-Meyer-Olkin (KMO) measure of sampling adequacy were applied. According to Tabachnick and Fidell [11], data is factorable when the KMO is above the minimum acceptable level of 0.60 . KMO values over 0.8 indicate that included variables are 'meritoriously' predicted without error by other variables. The KMO value of the variables was 0.896 , which indicated sampling adequacy such that the values in the matrix were sufficiently distributed to conduct factor analysis. The value obtained by Bartlett's test of sphericity, $\chi^{2}(55)$ was 16804.95, which was highly significant at $p<0.001$ level, indicating that the data were approximately multivariate normal [13].

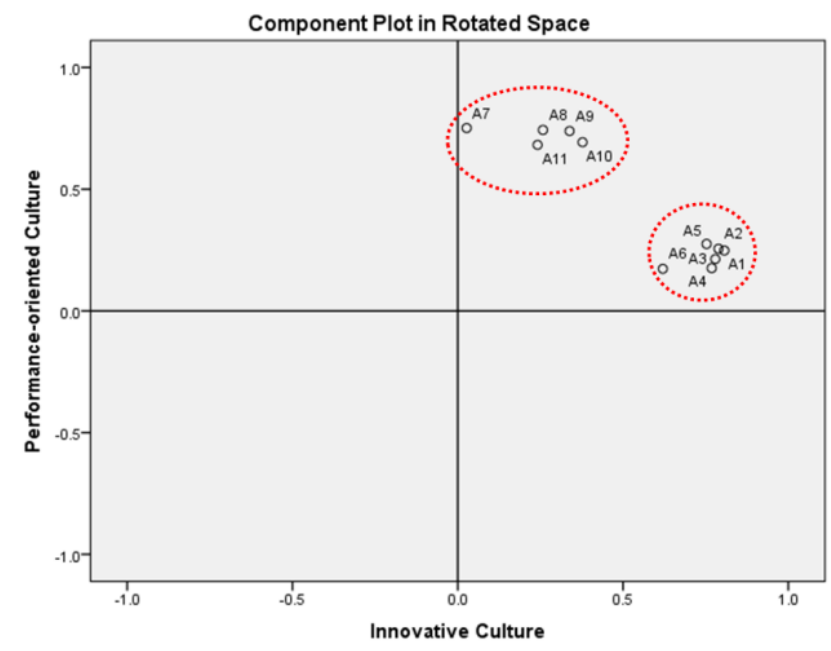

Fig. 1. A geometrical representation of the PAO construct

A principal component analysis (PCA) with varimax rotation was initially conducted to examine the validity of the construct. The goal of PCA is to extract the maximum variance from the data set with each component. A total of eleven variables were selected to operationally define the ACI construct. The presence of two components with eigenvalues greater than 1 was indicated by the initial principal 
component analysis. A geometrical approach can be utilised by the EFA where factors in a coordinate system can be visualized by variables plotted on the axes of a graph [14]. When the coordinates of variables are in close proximity to each graph, this represents the strength of the relationship between that variable and each factor. The variables were plotted as a function of the factors, as shown in Fig. 1. Six variables (A1, A2, A3, .., A6) have high factor loadings (i.e., a strong relationship) with factor 1 (innovative culture: horizontal axis) but have a low correlation with factor 2 (performance-oriented culture: vertical axis). In comparison, five variables have strong relationships with performance-oriented culture but low correlation with innovative culture.

The Catell's scree test employs a graphical plot of the eigenvalue of the factor in their order of extraction in which a sudden change of slope in the graph indicates the maximum number of factors to be extracted and determines the number of factors to retain. A horizontal and a vertical line starting at each end of the curve were inserted to determine whether an abrupt change of slope had occurred. Examination of the scree plot indicated that a sudden change of slope occurred after the second component (See Fig. 2).

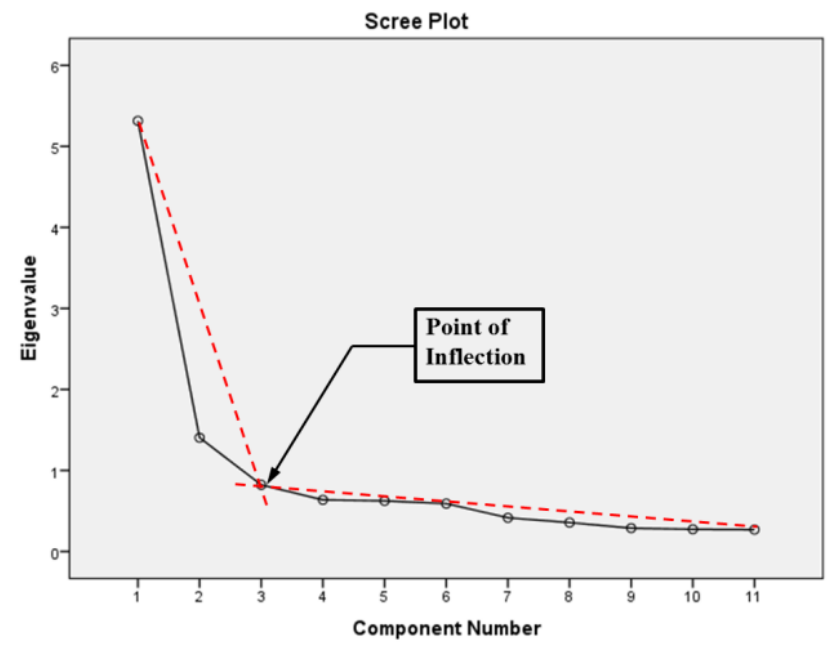

Fig. 2. Scree plot of principal component of the PAO construct

Prior to extracting factors, communality estimates must be generated. Communality is the proportion of observed variance accounted for by the common factors. These values represent the total amount of variance for an item explained by the extracted factors. The communality is denoted by $h^{2}$ and is the summation of the squared factor loadings of a variable across factor [11]. Generally, a variable is excluded from the analysis if it has low communalities (less than 0.20), which means that $80 \%$ is unique variance. This is because the objective of factor analysis is to describe the variance through the common factors. The formula for deriving the communalities is: 


$$
h_{j}^{2}=a_{j 1}^{2}+a_{j 2}^{2} \cdots \cdots+a_{j m}^{2}
$$

Where $a$ equals the loadings for $j$ variables.

Parallel analysis was also conducted for factor extraction, in which the eigenvalues derived from actual data were compared with the eigenvalues resulting from the random data. Factors are retained when actual eigenvalues exceed random data. SPSS syntax, as depicted in Fig. 3, (adapted from O' Connor [15]) can be used to achieve this by simply specifying the number of cases, variables, data sets, and the desired percentile for the analysis at the beginning of the program.

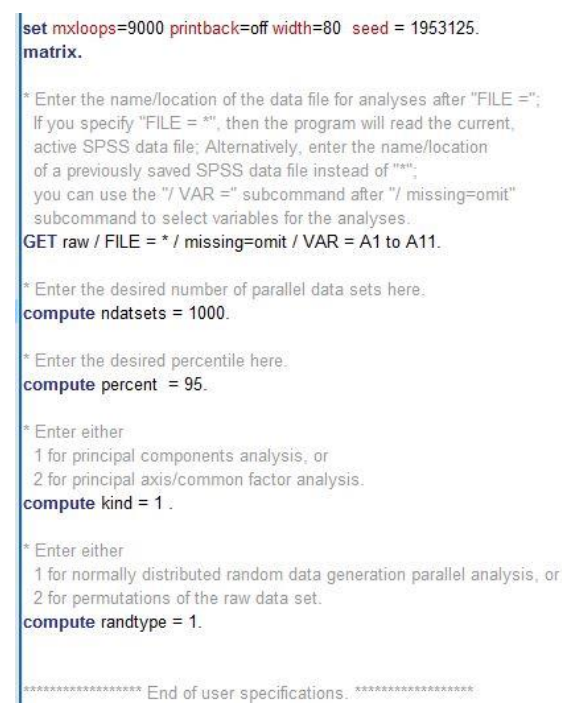

Fig. 3. SPSS Syntax for Parallel Analysis

The results, as shown in Table 1, indicate that only the first 2 actual eigenvalues are greater than those generated by random data, and therefore, these would be retained.

Table 1. Parallel Analysis

\begin{tabular}{cccc}
\hline \hline $\begin{array}{c}\text { Component } \\
\text { number }\end{array}$ & $\begin{array}{l}\text { Eigenvalues derived from } \\
\text { actual data }\end{array}$ & $\begin{array}{l}\text { Eigenvalues derived from } \\
\text { random data }\end{array}$ & Decision \\
\hline 1 & 5.315 & 1.094 & Accept \\
2 & 1.404 & 1.068 & Accept \\
3 & 0.823 & 1.049 & Reject \\
4 & 0.637 & 1.032 & Reject \\
\hline \hline
\end{tabular}

During the operationalisation, the structure of the data for the ACI construct was classified into two factors: innovative culture and performance-oriented culture. To date, no study has developed a framework to provide measurement variables for these 
two factors. The EFA was employed to empirically examine if the variables for the ACI construct could indeed be represented by these two factors. The initial principal component analysis indicated the presence of two components with eigenvalues greater than 1, which explained 61.1 percent of the total variance.

Using the factor loadings in Table 2, the communality of variable A1 was calculated using the aforementioned formula:

$$
h_{A 1}^{2}=0.806^{2}+0.248^{2}=0.712
$$

Communalities ranged from 0.415 to 0.712 , indicating relatively strong data. The patterns for the rotated factor loading, presented in Table 4 , revealed that all variables were well above the 0.50 threshold level. Moreover, the patterns for the loading of the variables onto their respective factors were also consistent with the operational definition of ACI construct in the literature review, assuring its robustness for this scale development. As with the original operationalisation, the ACI construct consisted of the following two factors:

- ACI1: Innovative culture (6 variables, component 1); and

- ACI2: Performance-oriented culture (5 variables, component 2).

Table 2. Rotated factor loadings of the ACI construct

\begin{tabular}{l|c|cc}
\hline \multicolumn{1}{c}{ Variable: Description } & \multicolumn{2}{c}{ Component } & \multirow{2}{*}{$\boldsymbol{h}^{2}$} \\
\cline { 2 - 3 } & \multicolumn{1}{c}{$\mathbf{1}$} & $\mathbf{2}$ & \\
\hline A1: My agency prioritises ideas development. & 0.81 & 0.25 & 0.71 \\
A2: Most managers encourage innovation. & 0.79 & 0.26 & 0.69 \\
A3: Internal communication is effective. & 0.78 & 0.21 & 0.65 \\
A4: Change is managed well in my agency. & 0.77 & 0.18 & 0.62 \\
A5: My agency emphasises innovation. & 0.75 & 0.28 & 0.64 \\
A6: My workplace provides access to learning. & 0.62 & 0.17 & 0.42 \\
A7: Managers make sure procedure is follow. & & & \\
A8: My agency emphasises task delivery. & 0.03 & 0.75 & 0.57 \\
A9: Most managers ensure their team delivers. & 0.26 & 0.74 & 0.62 \\
A10: My agency prioritises goals achievement. & 0.34 & 0.74 & 0.66 \\
\hline A11: My agency emphasises standardised services. & 0.38 & 0.69 & 0.62 \\
\hline \hline
\end{tabular}




\subsection{Confirmatory Factor Analysis}

Confirmatory factor analysis (CFA) is a method which enables evaluations of how well these measured variables represent the latent constructs. Although similar to exploratory factor analysis (EFA), but CFA differs in the sense that the number of constructs and indicators need to be specified before the computation of results. As such, it is considered as a "confirmatory" method [16]. CFA was conducted to examine whether these dimensions contributed to an overall construct of ambidextrous culture for innovation. To analyse the data using CFA, the Analysis of Moment Structures (AMOS) version 22 was employed to allow the data from an SPSS analysis set to be directly used in the AMOS calculation [17]. A 10-item measure of the two ACI dimensions derived from EFA was tested in AMOS. The results of the CFA conducted on AMOS have been presented in Fig 4. For the model to be considered as having an acceptable fit, all eight indices were measured against the criteria: GFI, AGFI, IFI, NFI, CFI and TLI > 0.90; RMSEA < 0.08; and RMR < 0.05 [1]. The initial fit indices were unsatisfactory. Examination of the modification indices provided clear evidence of misspecified error covariance associated with variables A3, A4, A7, and A9, thus they were removed from the model. The likelihood ratio test revealed yielded a $\chi^{2}$ of 129.12 and $d f=13$. The respecified model showed evidence of satisfactory model fit: GFI $=0.99$, AGFI $=0.98$, RMSEA $=0.05, \mathrm{RMR}=0.02, \mathrm{IFI}=0.99, \mathrm{NFI}=0.99, \mathrm{CFI}=0.99$, and $\mathrm{TLI}=0.98$.

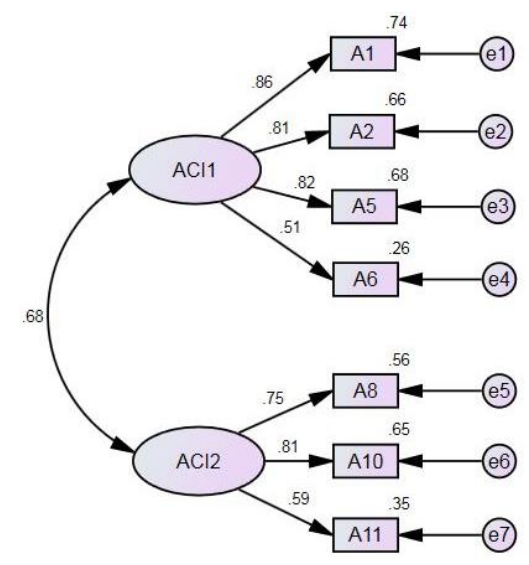

Fig. 4. CFA model of ACI construct

All of the variables loaded significantly $(p<0.001)$ on their respective constructs, as evidenced by the loading being greater than the threshold level of 0.50 (ranging from 0.51 to 0.86 ), indicating convergent validity. Almost all the variables had $R^{2}$ values greater than 0.50 . The variables with low $R^{2}$ values were retained since their loadings were substantial and highly significant. The correlation coefficient between the two factors was 0.68 . Thus, being less than 0.85 , discriminant validity was confirmed. 
Composite reliability (CR) measures the internal reliability of all the variables in their measurement of a construct. An average variance extracted (AVE) determines the amount of variance in the measured indicators captured by the latent construct. Bagozzi and Yi [18] suggested 0.60 and 0.50 as the minimum value of composite reliability and average variance extracted, respectively. CR and AVE values were calculated using the following equations [1]:

$$
\begin{aligned}
& C R=\frac{\left(\sum \lambda\right)^{2}}{\left(\sum \lambda\right)^{2}+\sum e} \\
& A V E=\frac{\sum \lambda^{2}}{\sum \lambda^{2}+\sum e}
\end{aligned}
$$

Where $\lambda$ is standardised factor loading and $e$ is the standardised error.

Table 3 summarises the reliability of each factor. Both factors have a Cronbach alpha greater than 0.70 , which shows measurement scales consisting of a set of homogeneous items to measure the meaning of the factor. Composite reliability for each factor in the CFA model was above 0.60, demonstrating that these factors had adequate internal consistency and were sufficient in their representation of the construct. Both factors also had AVE values greater than 0.50 , indicating that more variance was captured by the variables within each factor and shared more variance in the factor than with the other factor. This also means that the construct relative to the amount of variance was due to measurement error.

Table 3. Reliability tests of the ACI construct

\begin{tabular}{lccc}
\hline \hline Factor & $\boldsymbol{\alpha}$ & $\mathbf{C R}$ & $\mathbf{A V E}$ \\
\hline Innovative Culture & 0.83 & 0.84 & 0.58 \\
Performance-oriented Culture & 0.75 & 0.80 & 0.52 \\
\hline \hline
\end{tabular}

The respecified two-factor model had the acceptable model fit indices suggesting that unidimensionality could be supported. To measure scale reliability, the study utilised 'Cronbach's alpha' which provided an indication of how consistent the responses were across items within the scale. The recalculated Cronbach's alpha value of 0.849 was indicative of the reliability of the scale.

\section{Conclusion}

The paper presented here aimed to increase both the understanding of culture for innovation in the Australian Public Service and to improve the construct measurement scale. Perspectives on culture for innovation were identified by exploratory factor analysis. Two factors were extracted from the 11 variables which were selected and 
grouped according to culture type. Thus, 11 variables with factor loadings ranging from 0.620 to 0.806 were retained. The two factors extracted as characterising ambidextrous culture for innovation (ACI) were comprised of innovative culture and performance-oriented culture.

To assess the reliability and validity of the ambidextrous culture for innovation construct, a confirmatory factor analysis was conducted using the maximum likelihood estimation method. Initially, the model did not present an acceptable level of fit because variables A3, A4, A7, and A9 were associated with relatively high modification indices. Thus, it was prudent to respecify the model with the elimination of these four variables. After the model re-specification process, the CFA model fit very well with the collected data and the relationships between the observed variables and latent variables were significant. This study also provides a reliable measurement scale to measure culture for innovation in the Australian Public Service.

The two-factor model was applied both descriptively and diagnostically. It presented a practical way to measure an ambidextrous culture for innovation, and could initially be used to establish a baseline level of ambidextrous culture for innovation. The empirical evidence shows ambidextrous culture for innovation is reflected by the combination of innovative culture and performance-oriented culture. These findings highlight the importance of embedding both practices into the fabric of a government's culture. This could be achieved by senior managers promoting an innovative culture by allowing errors, encouraging employees to put forward their ideas, and cultivating a learning environment. Leaders should also facilitate a performance-oriented culture by supervising goal attainment, establishing standardised process, and adhering to plans. If successfully adopted, an ambidextrous culture for innovation will increase productivity, and may eventually result in a high performance organisation (HPO).

The validity and reliability of the construct were confirmed in the EFA and CFA, however it was still necessary to investigate for a common method bias. By taking into account the recommendations of Podsakoff et al. [19], Harman's single-factor test was conducted to assess the common method bias in this single setting design. As the results indicated no single factor underlying the data, the common method bias did not seem to exist.

\section{References}

1. Hair, J.F., et al., Multivariate data analysis: A global perspective. 2010, Basım Pearson Education.

2. Gerbing, D.W. and J.C. Anderson, An updated paradigm for scale development incorporating unidimensionality and its assessment. Journal of marketing research, 1988: p. 186-192.

3. Kline, R.B., Principles and practice of structural equation modeling. 2015, New York, NY: Guilford publications. 
4. Zhou, K.Z., et al., Developing strategic orientation in China: antecedents and consequences of market and innovation orientations. Journal of Business Research, 2005. 58(8): p. 1049-1058.

5. Lægreid, P., P.G. Roness, and K. Verhoest, Explaining the innovative culture and activities of state agencies. Organization Studies, 2011. 32(10): p. 13211347.

6. Vartanian, T.P., Secondary data analysis. 2010, New York, NY: Oxford University Press.

7. Brown, R.B. and M.P. Saunders, Dealing with statistics: what you need to know. 2007: McGraw-Hill Education (UK).

8. Frankfort-Nachmias, C. and D. Nachmias, Research methods in the social sciences. 7th ed. 2008, New York, NY: Worth.

9. Torugsa, N. and A. Arundel, Complexity of Innovation in the public sector: A workgroup-level analysis of related factors and outcomes. Public Management Review, 2016. 18(3): p. 392-416.

10. Torugsa, N.A. and A. Arundel, The nature and incidence of workgroup innovation in the Australian public sector: evidence from the Australian 2011 state of the service survey. Australian Journal of Public Administration, 2015.

11. Tabachnick, B.G. and L.S. Fidell, Using multivariate statistics. 2007, Needham Height, MA: Allyn \& Bacon.

12. Russell, D.W., In search of underlying dimensions: The use (and abuse) of factor analysis in Personality and Social Psychology Bulletin. Personality and Social Psychology Bulletin, 2002. 28(12): p. 1629-1646.

13. Pallant, J., SPSS survival manual: a step by step guide to data analysis using IBM SPSS. 2013, Maidenhead, UK: McGraw-Hill.

14. Field, A., Discovering statistics using IBM SPSS statistics. 4th ed. 2013, London: SAGE Publications.

15. O'connor, B.P., SPSS and SAS programs for determining the number of components using parallel analysis and Velicer's MAP test. Behavior Research Methods, Instruments, \& Computers, 2000. 32(3): p. 396-402.

16. Gallagher, D., L. Ting, and A. Palmer, A journey into the unknown; taking the fear out of structural equation modeling with AMOS for the first-time user. The Marketing Review, 2008. 8(3): p. 255-275.

17. Byrne, B.M., Structural equation modeling with AMOS: Basic concepts, applications, and programming. 2013, New York: Routledge.

18. Bagozzi, R.P. and Y. Yi, On the evaluation of structural equation models. Journal of the Academy of Marketing Science, 1988. 16(1): p. 74-94.

19. Podsakoff, P.M., et al., Common method biases in behavioral research: a critical review of the literature and recommended remedies. Journal of applied psychology, 2003. 88(5): p. 879. 